\title{
Correction to: Factors affecting occupational hazards among operating room personnel at hospitals Affiliated in Western Iran: a cross-sectional study
}

\author{
Javad Yoosefi Lebni ${ }^{1}$ - Farbod Ebadi Azar ${ }^{2} \cdot$ Manoj Sharma $^{3}$ - Alireza Zangeneh ${ }^{4}$. Neda Kianipour ${ }^{5}$. \\ Seyyed Amar Azizi ${ }^{6} \cdot$ Amir Jalali $^{7} \cdot$ Arash Ziapour $^{1}$
}

Published online: 30 June 2020

(C) Springer-Verlag GmbH Germany, part of Springer Nature 2020

\section{Correction to: J Public Health (Berl.) https://doi.org/10.1007/s10389-019-01169-y}

The affiliations of Seyyed Amar Azizi and Arash Ziapour are incorrect. It should be as shown below.

The online version of the original article can be found at https://doi.org/ 10.1007/s10389-019-01169-y

\author{
Arash Ziapour \\ arashziapoor@gmail.com \\ Javad Yoosefi Lebni \\ j.yousefi28@yahoo.com \\ Farbod Ebadi Azar \\ f_ebadi@yahoo.com \\ Manoj Sharma \\ manoj.sharma@jsums.edu \\ Alireza Zangeneh \\ ali.zangeneh88@gmail.com \\ Neda Kianipour \\ Nedakianipour@gmail.com \\ Seyyed Amar Azizi \\ amarazizi59@gmail.com \\ Amir Jalali \\ jalali_amir@yahoo.com
}

Health Education and Health Promotion, Health Institute, Kermanshah University of Medical Sciences, Kermanshah, Iran

2 Department of Health Services and Health Education, School of Health, Iran University of Medical Sciences, Tehran, Iran

3 Behavioral \& Environmental Health, School of Public Health, Jackson State University, Jackson, MS, USA

4 Social Development and Health Promotion Research Center, Health Institute, Kermanshah University of Medical Sciences, Kermanshah, Iran

5 Students Research Committee, Kermanshah University of Medical Sciences, Kermanshah, Iran

6 Health Institute, Kermanshah University of Medical Sciences, Kermanshah, Iran

7 Department of Psychiatric Nursing, School of Nursing and Midwifery, Substance Abuse Prevention Research Center, Kermanshah University of Medical Sciences, Kermanshah, Iran 